\title{
A Study on Assessment of Endothelial Dysfunction in Hypertension
}

\author{
Samir Kumar Panda ${ }^{1}$, Mrudula Vandana ${ }^{2}$, V.V.N. Goutham ${ }^{3}$ \\ ${ }^{1}$ Associate Professor, Department of General Medicine, ${ }^{2}$ Assistant Professor, Department of Radio-Diagnosis, ${ }^{3}$ Assistant \\ Professor, Department of General Medicine, GIMSR, Visakhapatnam, Andhra Pradesh, India \\ Corresponding author: Dr. Mrudula Vandana, w/o Dr. V.V.N. Goutham, Dno:45-58-5/1/1, Triveni Residency, Narasimha \\ nagar, Visakhapatnam-530024, Andhra Pradesh, India
}

DOI: http://dx.doi.org/10.21276/ijcmsr.2020.5.1.6

(c) BY-NC-ND

How to cite this article: Samir Kumar Panda, Mrudula Vandana, V.V.N. Goutham. A study on assessment of endothelial dysfunction in hypertension. International Journal of Contemporary Medicine Surgery and Radiology. 2020;5(1):A26-A30.

\section{A B S T R A C T}

Introduction: Endothelial Dysfunction(ED), well documented in essential hypertension, is produced by oxidation stress, breakdown and inactivation of NO, release of Endothelium D erivedConstricting Factor, prostanoids, endothelin - 1 leading to decreased bioavailability of NO sustained SBP and high mean BP blunts Flow Mediated Dilatation (FMD) response in brachial artery. Hence present study has been designed to measure flow mediated vasodilatation of brachial artery by high resolution ultrasonographic imaging and pulse Doppler study.

Material and Methods: This study has been carried out in the Department Of General Medicine \& Radio-Diagnosis during the session August 2018 to December 2019, in GIMSR, Visakhapatnam,Andhra Pradesh.Fifty hypertensive patients diagnosed as per JNC-VIII recommendation have been studied. Equal number of 50 healthy persons as 30 male and 20 female in the age group of 40-70 have been taken as control for comparison. Evaluation of their endothelial function was done in terms Resting Brachial Artery Diameter (RBAD)and FMD\% in hypertension group.

Results: RBAD was found to be $4.07 \pm 0.28$ in control, $3.98 \pm 3.4$ in hypertension group $(n=31)$ where SBP $>160 \mathrm{mmHg}$. 4.15 $\pm 0.37 \mathrm{~mm}$ (SBP 140-159 mmHg). Correlated with DBP the RBAD data were 90-109=3.84 $\pm 0.34,>=100=3.88 \pm 0.32 . \mathrm{HBAD}$ values in study group in relation to SBP and DBP showed:SBP $140-159=3.95 \pm 0.33, \mathrm{DBP} 90-109=4.08 \pm 0.37, \mathrm{SBP}>160=$ $4.15 \pm 0.37$ and DBP $>110=4.02 \pm 0.7$. FMD\% (flow Mediated dilation) is designated as an endothelial dependant process reflecting relaxation of an artery in response to increase flow and shear stress. Hyperemia is associated with increased blood flow, velocity and diameter.

Conclusion: FMD\% may not be useful for therapeutic purpose because large number of physiological factor alters this. It is a functional bioassay for, in vivo, endothelial function in human.Keywords: Flow-mediated dilatation,Brachial artery diameter,endothelial dysfunction, hypertensive

Keywords: Endothelial Dysfunction, Hypertension

\section{INTRODUCTION}

The vascular endothelium is a large paracrine organ that secretes numerous factors regulating vascular tone, cell growth, platelet and leukocyte interaction and thromobogenicity (Sun D. et al). Endothelium the special, single layer of cells that lines the cavities of heart, blood vessel, lymph vessel and serous cavity of the body. This single layer of cells line the interior surface of blood vessel, forming an interface between circulating blood in the lumen and the rest of the vessel wall. Endothelial cells line the entire circulatory system from heart to small capillaries. These cells reduce the friction of the flow of blood allowing distal propulsion of blood.

Endothelial cells have multifaceted role in vascular biology including:

1. Vasoconstriction and vasodilation and hence control the blood pressure.

2. Blood clotting (thrombosis and fibrinolysis)
3. Atherosclerosis

4. Formation of new blood vessel (angiogenesis)

5. Inflammation and edema.

Endothelial cells also control the exchange of blood cells, micronutrients and electrolytes. Thus, it is a crucial component of vascular structure, not only because it serves as barrier between flowing blood and vascular wall but also because it regulates vascular tone by releasing EDRF like Nitric oxide (NO).

In some organs, there are highly differentiated endothelial cells to perform specialized "filtering" functions. Example of such unique endothelial structures includes the renal glomerulus and blood brain barrier.

Endothelial dysfunction or the loss of proper endothelial function is a hallmark for vascular disease and often leads to atherosclerosis. Endothelial dysfunction is very common. Several chronic pathophysiologic conditions like Diabetes 
mellitus, Hypertension and coronary artery disease (CAD). One of the main mechanisms of endothelial dysfunction is the reduction of Nitric oxide (NO), often due to increased levels of asymmetric dimethylarginine which interface with the normal L-Arginine stimulated nitric oxide (NO) synthesis.

Blood vessel tone is determined by both the smooth muscle and endothelial function. Nitric oxide (NO) synthesized by vascular endothelium; relaxes the smooth muscle cells; inhibit platelet aggregation and cell proliferation. Thus vascular endothelial dysfunction has been implicated in the pathophysiology of several cardiovascular diseases including essential hypertension. Compromised vasodilatation due to endothelial dysfunction is mediated by impairment of release of factors that control the vascular tone.

Methods for assessment of endothelial dysfunction:

1. FMD (Flow Mediated Dilation) of brachial artery.

2. VOP (Venous Occlusion Plethysmography).

3. Iontophoresis with LDI (Laser Doppler imaging).

4. PWA (Pulse wave Analysis) for PWV (Pulse Wave Velocity).

5. MRI of brachial artery and coronary artery.

FMD of brachial artery, pulse wave velocity and carotid IMT (Intima Media Thickness) are examples of preferred noninvasive technique to detect cardiovascular risk and all these are used as a surrogate marker of artherosclerosis. PWV (Pulse Wave Velocity) which reflect arterial distensibility is measured by pressure volume pulse wave analysis, using a transducer. Carotid IMT measured by B-Mode ultrasonography detects morphologic and structural change in carotid artery.

Flow mediated dilation is designated as an endothelium dependant process, that reflects the relaxation of artery, when exposed to increase flow and there by increased sheer stress. This Physiologic process, first described by Schetzenmayer. Subsequently FMD was demonstrated in difficult arteries, most important of them being brachial artery.

This non-invasive technique for testing endothelial function by USG measurement of flow mediated dilation has recently generated considerable interest as a marker of atherosclerosis, played important role in prediction of clinical hypertensive and coronary events, and is found to be useful access to endothelial dysfunction. In human with abnormal endothelium dependant vasodilatation has been observed in the brachial artery. Patient with cardiovascular risk factors like smoking, hyperlipidemia, hypertension and diabetes seem to be associated with abnormal endothelial dysfunction and vasodilatations.

Although the use of angiographs and phase contrast magnetic resonance imaging for measuring flow mediated vascular dilatation has bee proposed. Ultrasonographic imaging continues to be the most commonly used method. Real time imaging low cost and simplicity without the need for image enhancing agent are some of its advantages.

Since endothelial function has been defined as an excellent barometer of vascular health disease process, it can be optimally utilized to gauge the severity of disease process like atherosclerosis and related pathophysiology. Hence present study has been designed to measure flow mediated vasodilatation of brachial artery by high resolution ultrasonographic imaging and pulse Doppler study.

\section{MATERIAL AND METHODS}

The present study has been undertaken in the department of General Medicine and in department of Radio-Diagnosis at GIMSR, Visakhapatnam, Andhra Pradesh during the period of August 2018 to December 2019.

Material of this study constitute of 50 healthy subjects taken as control and 50 patients of hypertension.

Patients attending medicine O.P.D., and diagnosed to have hypertension basing on the following criteria laid down in Eighth joint national committee, were taken.

After obtaining informed consent as per ethical committee recommendations selected cases were admitted to medical wards.

Detailed history as per proforma, through clinical examination supported by necessary laboratory tests and imaging studies were carried out as follows: Bio-chemical tests like - Lipid profile (TC, HDL), BMI, others like Chest X-Ray P/A View, ECG, fundoscopic examination, $\mathrm{CBC}$ and other routine examinations were done.

In both control and study group, endothelial dysfunction was evaluated by measurement of brachial artery flow mediated dilatation.

\section{Exclusion criteria}

Patients of diabetes mellitus, morbid obesity, coronary artery disease (CAD), cerobrovascular accidents (CVA), subarachnoid hemorrhage (SAH), and hypertensive emergensies are excluded from the study.

\section{Methodology}

Ethical committee clearance and informed consent from each patient was taken, patient was admitted and was instructed to abstain from alcohol, smoking caffeine and food for eight hours. He was then done taken to the department of Radiology and asked to lie quietly supine position of ten minutes. Then resting or base line diameter of right brachial artery was measured by B-Mode high resolution USG Doppler system. Brachial artery was scanned in longitudinal section by placing the transducer in antecubital fossa, three centimeter above the elbow. After basal recording, forearm ischmia was induced by inflating the pneumatic cuff and following reactivate hyperemia again brachial artery diameter was measured.

In addition to blood pressure recording, ECG and biochemical parameters required for diagnosis and inclusion of cases, special tests for endothelial dysfunction includes high resolution USG, pulse Doppler system.

Care was taken to identify exact centre of brachial artery in monitor after visualizing clearest picture of anterior and posterior intimal layer by proper positioning of transducer and pulse Doppler signal at an angle of 70 degree to the vessel with range gate $(1.5 \mathrm{~mm})$ in the centre of artery. Resting Brachial Artery Diameter (RBAD) was measured and recorded. Pneumatic cuff was tied after the right forearm was inflated to $250 \mathrm{mmHg}$ maintained for 4.5 minutes, then released after 60 seconds of deflation post hyperemic brachial artery diameter (HBAD) was again recorded. 
All data was collected from control and study group consisting of lipid profile, BMI, SBP and DBP and hyperemic brachial artery diameter were analyzed in tabular form and correlated.

\section{RESULTS}

The number of males in the age group of $40-50,51-60$ and $61-70$ are $13(26 \%), 6(12 \%)$ and $11(22 \%)$ respectively. The corresponding number of females being $10(20 \%), 4(8 \%)$ and $6(12 \%)$ respectively.

The number of males in the control group according to sex of age groups $40-50,51-60$ and $61-70$ are 20(40\%), $7(14 \%)$ and $1(2 \%)$ respectively. The corresponding number of females being $11(22 \%), 10(20 \%)$ and $1(2 \%)$ respectively.

\begin{tabular}{|l|c|c|}
\hline \multicolumn{3}{|c|}{ Control group } \\
\hline SBP & No. of controls & FMD\% \\
\hline $101-110$ & 3 & 13.57 \\
\hline $111-120$ & 21 & 12.49 \\
\hline $121-130$ & 26 & 12.58 \\
\hline \multicolumn{3}{|c|}{ Cases group } \\
\hline SBP & No. of cases & FMD\% \\
\hline $131-140$ & 0 & 0 \\
\hline $141-150$ & 6 & 6.15 \\
\hline $151-160$ & 17 & 6.74 \\
\hline $161-170$ & 9 & 5.56 \\
\hline $171-180$ & 14 & 5.62 \\
\hline $181-190$ & 4 & 5.02 \\
\hline \multicolumn{2}{|c|}{ Table-1: Relationship of SBP with flow mediated dilation } \\
(FMD\%) in control group and cases group \\
\hline
\end{tabular}

\begin{tabular}{|l|c|c|}
\hline \multicolumn{3}{|c|}{ Control group } \\
\hline DBP & $\begin{array}{c}\text { No. of controls } \\
(\mathbf{n}=5 \mathbf{0})\end{array}$ & FMD\% \\
\hline $60-70$ & 8 & 12.06 \\
\hline $71-80$ & 30 & 12.76 \\
\hline $81-90$ & 12 & 12.55 \\
\hline \multicolumn{3}{|c|}{ Cases Group } \\
\hline DBP & $\begin{array}{c}\text { No. of cases } \\
(\mathbf{n}=\mathbf{5 0})\end{array}$ \\
\hline $91-100$ & 7 & FMD\% \\
\hline $101-110$ & 41 & 6.77 \\
\hline $111-120$ & 2 & 3.10 \\
\hline Table-2: Relationship of DBP with flow mediated dilation \\
(FMD\%) in control group \& cases group \\
\hline
\end{tabular}

\begin{tabular}{|l|c|c|c|}
\hline SBP $(\mathbf{m m H g})$ & No. of cases & RBAD $(\mathbf{m m})$ & HBAD $(\mathbf{m m})$ \\
\hline$<140$ & 50 & $4.07 \pm 0.28$ & $4.57 \pm 0.33$ \\
\hline $140-159$ & 19 & $3.71 \pm 0.29$ & $3.95 \pm 0.33$ \\
\hline$>=160$ & 31 & $3.93 \pm 0.34$ & $4.15 \pm 0.37$ \\
\hline
\end{tabular}

Table-3: Systolic blood pressure with RBAD and HBAD

\begin{tabular}{|l|c|c|c|}
\hline $\begin{array}{l}\text { DBP } \\
(\mathbf{m m H g})\end{array}$ & No. of cases & RBAD $(\mathbf{m m})$ & HBAD $(\mathbf{m m})$ \\
\hline$<90$ & 50 & $4.07 \pm 0.28$ & $4.57 \pm 0.33$ \\
\hline $90-109$ & 44 & $3.84 \pm 0.34$ & $4.08 \pm 0.37$ \\
\hline$>=110$ & 6 & $3.88 \pm 0.32$ & $4.02 \pm 0.37$ \\
\hline \multicolumn{2}{|r|}{ Table-4: Diastolic blood pressure with RBAD and HBAD } \\
\hline
\end{tabular}

The table- 1 shows distribution of FMD\% in control group having SBP from 101-110, 111-120 and 121-130 are $13.57 \%, 12.49 \%$ and $12.58 \%$ respectively and distribution of FMD\% in cases group having SBP from 131-141, 141-150, $151-160,161-170,171-180$ and $181-190$ are $6.15 \%, 6.74 \%$, $5.56 \%, 5.62 \%, 5.02 \%$ respectively.

The table-2 shows distribution of FMD\% in controls having diastolic blood pressure ranges from 60-70, 71-80 and 81-90 are $12.06 \%, 12.76 \%$ and $12.55 \%$ respectively and distribution of FMD\% in cases having diastolic blood pressure ranges from $91-100,101-110$ and $111-120$ are $5.77 \%, 6.19 \%$ and $3.10 \%$ respectively.

The table-3 shows correlation of resting brachial artery diameter and ischemic induced brachial artery diameter with systolic blood pressure.

The table- 4 shows correlation of resting brachial artery diameter and ischemic induced brachial artery diameter with diastolic blood pressure.

\section{DISCUSSION}

In this study of hypertension patient of age group of 40 - 70 have been included, $26 \%$ of males and $20 \%$ of females are in $4^{\text {th }}$ decade and male preponderance $(60 \%)$ is observed (30 $\mathrm{M}: 20 \mathrm{~F})$.

Importance of age and sex in FMD\% determination cannot be over looked because advance in age sheer stress in across brachial artery changes leads to decrease $\mathrm{FMD} \%$. Simultaneously post menopausal women and others who are on OC pills have low FMD\%

SBP: Observation on hypertensive patients over a wide range of SBP from $131-190 \mathrm{mmHg}$ shows gradual decline in FMD\% with increase of SBP. In $151-160 \mathrm{mmHg}$, SBP range, FMD\% was 6.74\%, whereas in $181-190 \mathrm{mmHg}$ SBP range it significantly decreases to $5.02 \%$.

DBP:Similarly, diastolic blood pressure range increasing from $91-100 \mathrm{mmHg}$ to $111-120 \mathrm{mmHg}$ shows proportional decrease in FMD\% from $5.77 \%$ to $3.10 \%$.

Prolonged sustained hypertension resulting in persistent shear stress rise blunts $\mathrm{FMD} \%$ in brachial artery and also coronary artery an is indexed by the degree of LVH. Hypertension and endothelial dysfunction are intricately related and whether hypertension causes endothelial dysfunction or endothelial dysfunction is followed by hypertension is not clear. In some cases, endothelial dysfunction may antedate hypertension and contributes for development of hypertension.

Framingham Heart Study failed to determine whether endothelial dysfunction is cause or consequence of hypertension. A third factor like arterial stiffness has been attributed to affect endothelial dysfunction and systolic blood pressure.

In essential hypertension; reduced endothelial vasodilatation is characterized by impaired NO availabilities. ${ }^{1}$ With established relationship between hypertension, CAD \& atherosclerosis with a common parameter like endothelial vasodilatation. Now it has become pertinent to search for antithypertensive therapy containing special feature to restore $\mathrm{NO}$ availability and there by counteract reactive hyperemia induced impairment of FMD\%.

Brachial artery dilation - RBAD \& HBAD In control group, 
resting brachial artery diameter (RBAD) is found to be $4.07 \pm 0.28$ which increases to $4.57 \pm 0.33$ after hyperemia (Hypermic Brachial artery Diameter). In Hypertensive group 31 patients with $\mathrm{SBP}>160 \mathrm{mmHg} \mathrm{RBAD}$ is $3.93 \pm$ $3.4 \mathrm{~mm}$ and HBAD is $4.15 \pm 0.37 \mathrm{~mm}$. In middle range of SBP (140 - $159 \mathrm{mmHg}) .19$ cases shared RBAD value 3.71 \pm 0.29 and $\mathrm{HBAD}$ value $3.95 \pm 0.33 \mathrm{~mm}$.

Similarly, for diastolic blood pressure, different ranges $(<=90$, $90-109,>-110)$ RBAD values are $4.07 \pm 0.28,3.84 \pm 0.34$, $3.88 \pm 0.32$ respectively and $\mathrm{HBAD}$ values are $4.57 \pm 0.33$, $4.08 \pm 0.37,4.02 \pm 0.37$ respectively.

Above data suggest that as systolic or diastolic blood pressure increase, both RBAD and HBAD decreases. Moreover, affect of ischemia induced hyperemia of brachial artery increases the brachial artery diameter. ${ }^{2}$

These variations are dependent on a complex cascade controlling endothelial function like endothelin- 1, No, Shear stress and hyperemia. ${ }^{3}$ Control and hypertensive group shows RBAD values $3.97 \pm 0.61 \mathrm{~mm}$ and $4.10 \pm 0.5$ $\mathrm{mm}$ whereas; $\mathrm{HBAD}$ values are $4.30 \pm 0.94$ and $4.42 \pm 0.54$ respectively.

This clarifies that resting diameter before intervention may be higher in hypertensive group as compared to control. Depending on severity and duration of hypertension and whether it is producing sustained shear stress or not. However, after ischemia, HBAD value may appear to be higher than control but practically net hyperemia dilatation and consequence $\mathrm{FMD} \%$ is found to be decreased in hypertensive group. ${ }^{4}$

\section{Brachial artery fmd model}

In present study, $\mathrm{FMD} \%$ has been evaluated in four groups like control $(n=50)$, hypertensive $(n=50)$, with LVH $(n=15)$ and hypertensive without LVH $(\mathrm{n}=35)$.

FMD\% in normal and hypertensive groups are $12.4 \pm 2.2 \%$ and $5.6 \pm 2.4 \%$ respectively. Whereas, FMD $\%$ in hypertension without $\mathrm{LVH}$ and with $\mathrm{LVH}$ are $6.8 \pm 3.6 \%$ and $3.4 \pm 1.8 \%$ respectively.

FMD\% value in control and hypertensive group shows statistically significant decrease (pvalue $=<.001$ ). Takeshi Moatayama et al; have reported $8.0 \pm 2.5 \%$ in control $\mathrm{Vs}_{\mathrm{s}}$ $5.6 \pm 3.0$ in hypertensive group. Their report value in LVH group and non LVH group are $2.9 \pm 2.6 \%$ and $4.2 \pm 1.8 \%$ respectively. ${ }^{5}$

Higher values of FMD\% encountered in present study may be explained by several factors influencing FMD\% measurement. Imortant factors which can be altered are:

- $\quad$ Time of test - 7 A.M to 9 A.M., fasting, abstinence from caffeine, smoking etc. ${ }^{6,7,8,9}$

- Site of cuff - distal or proximal, i.e., either $5 \mathrm{~cm}$ above or below elbow.

- Exact determination of site of transducer (at an angel of $70^{\circ}$ to center of artery.

- Proper identification of center of blood column in brachial artery.

- Pressure and duration of cuff inflation -

- $220 \mathrm{mmHg}$ for 4'

- $250 \mathrm{mmHg}$ for $4.5^{\prime}$

- $300 \mathrm{mmHg}$ for 5 ,
- After release of cuff, scanning is started at 60 seconds. Alternately, the first scan is taken at 45 seconds, 60 seconds, 90 seconds or continuous scan from 30 seconds before release to 90 seconds after deflation of cuff.

- Such continuous scanning over a prolonged period would be highly informative for flow velocity accuracy.

- Transducer should be maintained in a fixed position without shaking in relation to patient's are.

- Proper identification of $m$ line (interface between media and adventitia from anterior to posterior at the end of diastole.

Since measurement of brachial artery diameter is procedure dependent, observe dependent and is influenced by all above parameters, variation in FMD\% projection by various study groups may be valid.

Significant decrease of FMD\% in hypertensive group compare to control $(5.6 \pm 2.4 \% \mathrm{Vs} 12.4 \pm 2.2 \%)$ reflects a series of events following forearm occlusion and pathophysiologic sequence occurring in brachial artery and its wall differently in both groups. ${ }^{10}$

During distal occlusion, velocity (V), diameter (D) and blood flow increases during hyperemia velocity and diameter increases. Velocity reaches maximum value in both hypertension and control. Diameter increases both group. Changes in diameter were positively and significantly correlated to change in velocity in hyperemia.

Although not widely accepted some studies have reported a reduction in reactive hyperemia in patients having cardiovascular risk factors like hypertension and CAD.

Impaired FMD\% can be related to old age, sex, OCP, insulin, resistance, obesity and hypertension. Reactive hyperemia is the stimulus for FMD\%. ${ }^{11}$

It can be postulated that described hyperemia leads to decrease stimulus. Shear stress computed as area under the curve contributes significantly to FMD\%.

Proposed Mechanisms for FMD

1. Superoxide mediated inactivation of NO. ${ }^{12}$

2. Exercise induced up regulation of eNOs. ${ }^{13}$

3. Endothelium derived Prostanoids (can be blocked by Indomethacin)

4. Hypoxia of smooth muscles

5. Altered myogenic Response.

FMD\% is a diagnostic aid for evaluation of endothelial function. It is an experimental tool to measure endothelial dysfunction which is fundamental basis of atherogensis and CAD. FMD\% may predict future cardio vascular events. It is a user friendly, non-invasive, cheap, reliable, reproducible technique for risk stratification of CAD. ${ }^{14,15}$

\section{CONCLUSION}

FMD\%may be used as screening test before cornory angiography. It is safe in pregnancy and for children. Till date this remains a research and experimental tool. For wider clinical application, this study requires standardization and stringent protocol and consistent analysis. There is need of a comprehensive project inclusive of FMD\%, blood flow, peak systolic flow velocity and Shear stress measurement for wider clinical application. Since most of the studies including ours have limitations of simply measuring FMD\% neglecting 
important parameters like blood flow and flow velocity and DSS results have been inconsistent and misleading. A comprehensive project consisting of FMD, flow velocity, blood flow and shear stress. Thus, longitudinal studies are required to know the utility of $\mathrm{ED}$ as predictor of $\mathrm{CAD}$ in Indian population.

\section{REFERENCES}

1. Cardiol 1995; 26:1235-1241. Bae JH. Evaluation of endothelial function J Cardiol. 2001; 37(1): 89-92

2. Corretti MC, Plotnick GD, Vogel RA: Techinical aspects of evaluating brachial artery endotheliumdependent vasodilation using high frequency ultrasound. Am J Physiol 1995; 268(5): H1397-H1404.

3. Anderson TJ, Uehata A, Gerhard MD, et al. Close relation of endothelial function in the human coronary and peripheral circulation. J Am Coll Cardiol 1995; 26(3):1235-1241

4. Correttie MC, Anderson TJ, Benjamin EJ, et al. Guidelines for the ultrasound assessment of endothelialdependent flow-mediated vasodilation fo the brachial artery

5. Motoyama T, Kawano Kugiyama K, et Flow-mediated, endothelium-dependent dilation of the brachial arteries is impaired in patients with coronary spastic anigina. Am Heart J 1997; 13(2):263-267

6. Corretti MC, Plotnick GD, Vogel RA. Smoking correlates with flow-mediated brachial artery vasoactivity but not cold pressor vasoactivity in men with coronary artery disease. Int J card. Imaging. 1998; 14(1):11-7.

7. Heitzer T, Just H, Miinzel T: Antioxidant vitamin C improves endothelial dysfunction in chronic smokes. Circulation 1996; 94(6):6-9

8. Lekakis J, Papamichacl C, Vemmos C, et al. Efect of acute cigarette smoking on endothelium-dependent brachial artery dilatation inhealthy individuals Am J Cardiol 197; 7(4):529-531.

9. Motoyama T, Kawano H, Kugiyama K, Hirashima 0, Ohgushi M, Yoshimura M, Ogawa H, Yasue $\mathrm{H}$ : endothelium-dependent vasodilation in the brachial artery is impaired in healthy smokers: Efect of vitamin C. AM J Physiol 198;273(1):H164-H1650.

10. Meredith IT, Curie KE, Anderson TJ, et al. Postischemi Vasodilation in human forearm is dependent on endothelium-derived nitric oxide Am J Physiol 196; 270(4): H1435- H1440

11. Frangi AF, Laclaustra M,Lamata P.A registration-based approach to quantity flow-mediated dilation (FMD) of the brachial artery in ultrasound image sequences. IEEE Trans Med Imaging 2003;22 (11):1458-69.

12. Fukai T, Siegfried MR, Ushino-Fukai M, et al. Regulation of the vascular extracellular superoxide disunitase by nitric oxide and excersice training. J Clin Invest 2000; 105(1):16311639

13. Grunfeld S Hamilton CA, Mesaros S, McClain SW, Dominiczak AF, Bohr DF, Malinski T: Role of superoxide in the depressed nitric oxide production by the endothelium of genetically hypertensive rats, Hypertension 1995; 26(3):854-857

14. Neuteufl ' 1 '. Katzenschlager ft, Hassan A, et al.
Systemic endothelial dysfunction is related to the extent and severity of coronary artery disease. Atherosclerosis 1997: 129(6):111-118

15. Schroeder. $\mathrm{S}$ et al. FMD as a screening test for CAD and predictive value, Am. Heart J. 1999, 138(1):731-739

Source of Support: Nil; Conflict of Interest: None

Submitted: 13-12-2019; Accepted: 07-01-2020; Published online: 17-01-2020 